\title{
Imagem corporal e risco para desenvolvimento de transtornos alimentares em alunos
}

\section{de Nutrição e Educação Física}

\author{
Body image and risk for development of eating disorders in Nutrition and Physical Education \\ students
}

Imagen corporal y riesgo de desarrollo de trastornos de la alimentación en estudiantes de Nutrición

\section{y Educación Física}

Recebido: 05/01/2022 | Revisado: 11/01/2022 | Aceito: 16/01/2022 | Publicado: 18/01/2022

\author{
Sarah Regina da Silva Lucena \\ ORCID: https://orcid.org/0000-0002-3341-5275 \\ Centro Universitário Cesmac, Brasil \\ E-mail: sarahlucena9@gmail.com \\ Isabela Braga Peixoto \\ ORCID: https://orcid.org/0000-0003-0647-156X \\ Centro Universitário Cesmac, Brasil \\ E-mail: Peixoto.isabela2@gmail.com \\ Ismaell Avelino de Sousa Sobrinho \\ ORCID: https://orcid.org/0000-0002-7937-1941 \\ Universidade Federal de Alagoas, Brasil \\ E-mail: ismaellavelinos@gmail.com \\ Rita de Cássia Tenório Nunes \\ ORCID: https://orcid.org/0000-0003-4629-1248 \\ Centro Universitário Cesmac, Brasil \\ E-mail: rita.tenorio.nunes@gmail.com \\ Olivia Maximiano de Oliveira Sillero \\ ORCID: https://orcid.org/0000-0002-7273-4914 \\ Centro Universitário Cesmac, Brasil \\ E-mail: olivia.sillero@gmail.com \\ Kandeia Barros Ribeiro \\ ORCID: https://orcid.org/0000-0001-8988-3580 \\ Centro Universitário Cesmac, Brasil \\ E-mail: kandeia_barros@hotmail.com \\ Ana Cléria Gonçalves de Carvalho \\ ORCID: https://orcid.org/0000-0002-3251-9785 \\ Centro Universitário Cesmac, Brasil \\ E-mail: anacleriagc@gmail.com \\ Isabele Rejane de Oliveira Maranhão Pureza \\ ORCID: https://orcid.org/0000-0002-0652-429X \\ Centro Universitário Cesmac, Brasil \\ E-mail: isabelemaranhaopureza@gmail.com \\ Giane Meyre de Assis Aquilino \\ ORCID: https://orcid.org/0000-0002-5408-8672 \\ Centro Universitário Cesmac, Brasil \\ E-mail: giaquilino@hotmail.com \\ Waléria Dantas Pereira Gusmão \\ ORCID: https://orcid.org/0000-0002-4549-1363 \\ Centro Universitário Cesmac, Brasil \\ E-mail: waeleriadantasnut@gmail.com
}

\begin{abstract}
Resumo
A insatisfação com a imagem corporal, a prevalência de formas corporais extremas e comportamentos de controle de peso estão aumentado entre estudantes universitários de ambos os sexos. Apesar de ainda serem mais frequentes em estudantes do sexo feminino, jovens e na formação universitária. Investigações apontam alta prevalência desses comportamentos em estudantes da área da saúde, especialmente da Nutrição e Educação Física. O presente trabalho teve como objeto avaliar a imagem corporal e o risco de desenvolvimento de transtornos alimentares em estudantes de Nutrição e Educação Física de uma instituição de ensino superior privada do Estado de Alagoas, levantando a problemática na comunidade acadêmica. Tratou-se de um estudo transversal, de natureza exploratória e descritiva, no qual pretendeu-se recrutar todos os estudantes devidamente matriculados dos períodos iniciais e finais dos cursos de
\end{abstract}


Nutrição e Educação Física, no período de 2019.2 e 2020.1. Foram incluídos 86 participantes, sendo 25 (29,06\%) participantes do sexo masculino e $61(70,93 \%)$ do sexo feminino. Os acadêmicos dos cursos de Nutrição iniciaram a graduação com maior tendência a um comportamento alimentar disfuncional e maior distorção de imagem, diminuindo com o decorrer dos anos de graduação; entre os graduandos em Educação Física ocorreu o inverso, com maior risco de distorção de imagem e transtornos alimentares nos discentes do fim do curso, o que pode ser considerado um fenômeno preocupante.

Palavras-chave: Insatisfação corporal; Imagem corporal; Transtornos da alimentação; Estudante; Nutrição; Educação Física.

\begin{abstract}
Body image dissatisfaction, the prevalence of extreme body shapes and weight control behaviors are increasing among college students of both sexes. Although they are still more frequent in female students, young people and in university education. Investigations show a high prevalence of these behaviors in students in the health field, especially in Nutrition and Physical Education. This study aimed to evaluate body image and the risk of developing eating disorders in Nutrition and Physical Education students from a private higher education institution in the State of Alagoas, raising the issue in the academic community. It was a cross-sectional study, exploratory and descriptive, in which the intention was to recruit all students duly enrolled in the initial and final periods of the Nutrition and Physical Education courses, in the period between 2019.2 and 2020.1. 86 participants were included, 25 (29.06\%) of whom were male and $61(70.93 \%)$ were female. The students of Nutrition courses started graduation with a greater tendency towards dysfunctional eating behavior and greater image distortion, decreasing over the years of graduation; among undergraduates in Physical Education, the opposite occurred, with a greater risk of image distortion and eating disorders in students at the end of the course, which can be considered a worrying phenomenon.
\end{abstract}

Keywords: Body dissatisfaction; Body image; Eating disorders; Students; Nutrition; Physical Education.

\title{
Resumen
}

La insatisfacción con la imagen corporal, la prevalencia de formas corporales extremas y las conductas de control de peso están aumentando entre los estudiantes universitarios de ambos sexos. Aunque todavía son más frecuentes en alumnas, jóvenes y en la enseñanza universitaria. Las investigaciones muestran una alta prevalencia de estos comportamientos en estudiantes del campo de la salud, especialmente en Nutrición y Educación Física. Este estudio tuvo como objetivo evaluar la imagen corporal y el riesgo de desarrollar trastornos alimentarios en estudiantes de Nutrición y Educación Física de una institución privada de educación superior en el estado de Alagoas, planteando el tema en la comunidad académica. Se trató de un estudio transversal, exploratorio y descriptivo, en el que la intención fue reclutar a todos los estudiantes debidamente matriculados en los períodos inicial y final de los cursos de Nutrición y Educación Física, en el período comprendido entre 2019.2 y 2020.1. Se incluyeron 86 participantes, de los cuales 25 $(29,06 \%)$ eran hombres y 61 (70,93\%) eran mujeres. Los alumnos de los cursos de Nutrición iniciaron la graduación con una mayor tendencia a conductas alimentarias disfuncionales y una mayor distorsión de la imagen, disminuyendo a lo largo de los años de graduación; entre los licenciados en Educación Física ocurrió lo contrario, con un mayor riesgo de distorsión de la imagen y trastornos alimentarios en los estudiantes al final del curso, lo que puede considerarse un fenómeno preocupante.

Palabras clave: Insatisfacción corporal; Imagen corporal; Trastornos de alimentación; Estudiantes; Nutrición; Educación Física.

\section{Introdução}

A imagem corporal, definida como a imagem psicológica individual, de sua aparência física, é qualquer emoção positiva ou negativa de uma pessoa sobre a forma e o tamanho do corpo (Momeni, Ghorbani, \& Arjeini, 2020). Envolvendo aspectos relacionados à aparência, à estrutura, além de outros componentes psicológicos e físicos, a imagem corporal é formada a partir de valores, de afetos, da própria história pessoal e das múltiplas influências sócio-histórico-culturais que recebem (Garcia, Castro \& Soares, 2010).

Antigamente, o modelo de beleza exaltado era de um corpo feminino voluptuoso. Contudo, com o passar dos anos, os padrões foram sofrendo transformações e passaram a ser constituídos, por uma figura de um corpo magro, esguio e atlético. Ao valorizar a forma do corpo, muitas vezes os aspectos de saúde acabam sendo desconsiderados, pois para se encaixar em padrões impostos pela sociedade muitas pessoas acabam desenvolvendo imagens corporais negativas, decorrentes de altos níveis de insatisfação corporal (Fernandes et al., 2021). Uma percepção equivocada da autoimagem, assim como um estado 
depressivo, pode aumentar o risco de transtornos alimentares (Zaccagni, Rinaldo, Bramanti, Mongillo, \& Gualdi-Russo, 2020).

Os transtornos alimentares (TA) são síndromes caracterizadas por modificações severas do comportamento alimentar, que exibem a maior mortalidade quando comparada aos outros transtornos psiquiátricos (American Psychiatric Association, 2014). Estes transtornos têm se destacado, clínica e epidemiologicamente, nas últimas décadas (Moraes et al., 2016). Os transtornos alimentares tendem a começar de forma leve, com atitudes anormais em relação à alimentação e nutrição, como relações atípicas em relação ao peso atual e ideal, concepção do corpo, comportamento alimentar e preocupações excessivas com a comida, com o metabolismo e com a excreção do corpo (Momeni, Ghorbani, \& Arjeini, 2020). Caso os transtornos alimentares progridam, estes podem ter consequências graves aos indivíduos, tanto psicológica quanto fisiologicamente, inclusive podendo pôr em risco a vida dos acometidos.

Embora existam diversos tipos de transtornos alimentares, eles estão intimamente relacionados por apresentarem psicopatologia comum, na qual se destacam: recusa em manter um peso corporal adequado para sua estatura, preocupação excessiva com o peso (medo de engordar) e distorção da imagem corporal, além da negação da condição patológica (Legnani et al., 2012), que levam os indivíduos a se engajarem em dietas extremamente restritivas ou a utilizarem métodos inapropriados para alcançarem o corpo idealizado. Tais pacientes costumam julgar a si mesmos quase que exclusivamente pela aparência física, sentindo-se sempre insatisfeitos (Penz, Bosco, \& Vieira, 2008). A anorexia e a bulimia são os transtornos alimentares mais graves e estão associados a quadros clínicos de desvios nutricionais, alterações metabólicas, gastrintestinais, endócrinas e renais (Mazzaia \& Santos, 2018).

A insatisfação com a imagem corporal e a prevalência de formas corporais extremas e comportamentos de controle de peso estão aumentado entre estudantes universitários de ambos os sexos. Apesar de ainda serem mais frequentes em estudantes do sexo feminino, jovens e na formação universitária. As mulheres tendem a mostrar um forte desejo por magreza e usam dietas e comportamentos de controle de alimentos para perder peso e alcançar a imagem corporal desejada (Ebrahim, Alkazemi, Zafar, \& Kubow, 2019). Esses comportamentos alimentares disfuncionais parecem ter uma forte correlação com a insatisfação corporal e o perfeccionismo do sexo feminino (He, Li, Liu, \& Ma, 2021).

Estudos envolvendo transtornos alimentares e a insatisfação com a imagem corporal têm se concentrado em jovens, especialmente universitários, pelo fato de serem considerados grupos de maior risco. Investigações apontam alta prevalência desses comportamentos em estudantes da área da saúde, especialmente da Nutrição e Educação Física, que serão futuros profissionais de uma área que sofre uma forte cobrança da sociedade, remetendo a uma preocupação constante com a aparência física e a boa forma (Moraes et al., 2016). Alguns estudos seccionais sobre anorexia nervosa, bulimia nervosa e transtornos de compulsão alimentar envolvendo estudantes universitários e da área da saúde vêm sendo realizados na última década, indicando prevalências muito elevadas variando de 33,3\% na área de Nutrição, 28,6\% em Psicologia e 12,2\% em Educação Física (Rocha et al., 2020). Em relação a imagem corporal, pesquisas identificaram uma prevalência de insatisfação em 78,2\% de acadêmicos que cursam Educação Física (Gonçalves, Barbosa, Rosa, \& Rodrigues, 2008).

É preocupante pensar que os profissionais que devem ser habilitados para educar e tratar indivíduos com problemas que envolvem peso e forma corporal sejam acometidos por esses problemas, uma vez que pode influenciar a prática profissional (Moraes et al., 2016). Há uma necessidade crescente de pesquisas que investiguem a prevalência de distorções de imagem, bem como de transtornos alimentares entre estudantes ao longo da graduação e se os conhecimentos adquiridos ao longo do curso podem influenciar no comportamento alimentar.

Diante do exposto, o presente trabalho avaliou a imagem corporal e o risco de desenvolvimento de transtornos alimentares em estudantes de Nutrição e Educação Física de uma instituição de ensino superior privada do Estado de Alagoas, levantando a problemática na comunidade acadêmica. 


\section{Material e Método}

Tratou-se de um estudo transversal, de natureza exploratória e descritiva (Hochman, Nahas, Oliveira \& Ferreira, 2005), no qual pretendeu-se recrutar todos os estudantes devidamente matriculados dos períodos iniciais e finais dos cursos de Nutrição e Educação Física, no período de 2019.2 e 2020.1, a previsão era da inclusão de aproximadamente 130 alunos de Nutrição e 135 alunos de Educação Física.

Os participantes da pesquisa estavam sendo recrutados por convite verbal e presencial nas salas de aula de uma instituição de ensino superior privada. Após a autorização da coordenação do curso de Nutrição e Educação Física, os pesquisadores esclareceram os objetivos do estudo aos acadêmicos de ambos os cursos, a finalidade do estudo, disponibilizaram o TCLE e os questionários; além de informar sobre a garantia do seu carácter confidencial, voluntário e liberdade de escolha na participação e nas respostas da pesquisa.

Entretanto, pouco tempo depois do início do estudo, houve o decreto da Organização Mundial de Saúde de pandemia pela Covid-19, em 11 de março de 2020, e decorrente suspensão das aulas presenciais, os participantes passaram a ser recrutados via mídias digitais, após aprovação de emenda encaminhada ao Comitê de Ética em Pesquisa. Caso o aluno se dispusesse a participar da pesquisa recebia todas as informações necessárias quanto à realização do estudo em todas as suas etapas, podendo desistir quando lhe aprouvesse. Como marco inicial da pesquisa o acadêmico, necessitou ler e assinar o Termo de Consentimento Livre e Esclarecido (TCLE), baseado nas diretrizes da resolução CNS/MS 466/12, concordando com a participação na pesquisa.

Foram incluídos os alunos devidamente matriculados nos cursos de Nutrição e Educação Física dos períodos iniciais e finais, de ambos os sexos e maiores de 18 anos. Os questionários utilizados para avaliar a imagem corporal e o risco de desenvolvimento de transtornos alimentares que precisavam ser respondidos pelos acadêmicos Nutrição e Educação Física foram:

1. Body Shape Questionnaire - que consiste em uma escala tipo Likert, traduzida e validada para o português, com 34 itens auto preenchíveis e construída com o objetivo de mensurar a preocupação com a forma corporal e com o peso. Cada questão apresenta seis possibilidades de resposta: nunca, raramente, às vezes, frequentemente, muito frequentemente e sempre. Por questões éticas, no questionário, foi acrescentada a opção de resposta "prefiro não responder"; pensando em minorar possíveis constrangimentos aos perticipantes da pesquisa. A distorção de imagem corporal pode ser, de acordo com o resultado desta escala, classificada como: leve (70 a 90 pontos), moderada (90 a 110 pontos) ou intensa (>110 pontos) (Cooper, Taylor, Cooper, \& Fairburn, 1987; Di Pietro \& Silveira, 2009).

2. Eating Attitudes Test (EAT-26) - que é um questionário de autopreenchimento, traduzido e validado para o português, composto por 26 questões na forma de escala Likert, com as seguintes respostas possíveis: sempre, muitas vezes, frequentemente, poucas vezes, quase nunca e nunca. Da mesma forma que o instrumento anterior, e pelo mesmo motivo, foi acrescentada a opção “prefiro não responder”. A questão 25 apresenta pontuação invertida, ou seja, as alternativas sempre, muitas vezes e frequentemente são avaliadas com pontuação reversa (Garner, Olmsted, Bohr \& Garfinkel, 1982). O escore total do questionário é calculado a partir da soma das respostas de cada item, variando de 0 a 78 pontos, sendo que quanto maior o escore, maior o risco de desenvolvimento de TA. Considera-se que escores maiores que 21 são indicativos de comportamento alimentar de risco para TAs (Garner, Olmsted, Bohr \& Garfinkel, 1982; Rivas, Bersabé, Jumenez, \& Berrocal, 2010). O questionário é composto por três subescalas, cada qual avaliando fatores distintos do comportamento alimentar: dieta (13 itens), bulimia e preocupação com alimentos (seis itens) e autocontrole oral (sete itens).

3. Escala de silhueta de Stunkard - são imagens corporais que geralmente variam de um sujeito muito magro a um obeso. O participante do estudo devia escolher qual figura o melhor representava (silhueta atual) e com qual gostaria de se 
parecer (silhueta desejada). Para diminuir possíveis constrangimentos aos acadêmicos, conforme é mandatório nas normas éticas, foi acrescentada a opção de resposta "prefiro não responder. A insatisfação corporal é representada pela discrepância entre essas medidas (Stunkard, Sorensen, \& Schulsinger, 1983; Kakeshita \& Almeida, 2006).

Após a consolidação da resposta dos acadêmicos dos cursos de Nutrição e Educação Física, as variáveis categóricas foram apresentadas como frequências relativas e absolutas. Para comparar a frequência de comportamentos de risco e de distorção da imagem corporal e os cursos foi realizado o teste qui-quadrado, na presença de mais de $20 \%$ das células com contingência esperada menor que 5 foi utilizado o teste exato de Fisher. Todas as análises foram conduzidas com auxílio do software estatístico SPSS.

\section{Resultados e Discussão}

Dos 130 alunos matriculados, nos anos inicias e finais, do curso de Nutrição, 52 concordaram em participar da pesquisa e dos 135 alunos matriculados em Educação Física, 34 concordaram em participar do estudo, totalizando 86 participantes, sendo 25 (29,06\%) participantes do sexo masculino e 61 (70,93\%) do sexo feminino.

A faixa etária dos estudantes variou dos 18 aos 48 anos. Conforme é possível observar na tabela 1, a média de idade dos alunos matriculados nos períodos iniciais do curso de nutrição é de 25,71 (+/- 3,36) anos e nos períodos finais do curso é de 24,52 (+/-3,43) anos. Ao se tratar dos valores de Índice de Massa Corpórea (IMC), a média foi de 22,60 (+/- 1,78) Kg/m² nos períodos iniciais e de 22,41 (+/-1,22) $\mathrm{Kg} / \mathrm{m}^{2}$ nos períodos finais. Sendo a maioria dos alunos classificados pelo parâmetro do IMC no limiar de eutrofia.

Com relação aos alunos matriculados no curso de educação física a média de idade foi de 22,25 (+/-3,24) anos para os alunos dos períodos iniciais e de 23,63 (+/-1,00) anos para os dos períodos finais. Referente aos valores de IMC foi possível observar a média de 24,21 (+/-2,42) $\mathrm{Kg} / \mathrm{m}^{2}$ nos períodos iniciais e de $26,29(+/-2,53) \mathrm{Kg} / \mathrm{m}^{2}$ nos períodos finais. De maneira geral, variando entre o limiar de eutrofia e sobrepeso, de acordo com a classificação do parâmetro do IMC.

Tabela 1. Caracterização dos acadêmicos do curso de nutrição e educação física, de acordo com os anos de curso de uma instituição de ensino superior privada de Alagoas, de julho de 2019 a junho de 2020.

\begin{tabular}{|c|c|c|c|c|}
\hline & \multicolumn{2}{|c|}{ CURSO DE NUTRIÇÃO } & \multicolumn{2}{|c|}{ CURSO DE EDUCAÇÃO FÍSICA } \\
\hline CARACTERIZAÇÃO & ANOS & ANOS & ANOS & ANOS \\
\hline DOS ALUNOS & INICIAIS & FINAIS & INICIAIS & FINAIS \\
\hline Idade (anos) & $25,71(+/-3,36)$ & $24,52(+/-3,43)$ & $22,25(+/-3,24)$ & $23,63(+/-1,00)$ \\
\hline Peso $(\mathrm{Kg})$ & $63,23(+/-5,57)$ & $59,18(+/-3,75)$ & $70,87(+/-10,19)$ & $73,58(+/-6,23)$ \\
\hline Altura (m) & $1,66(+/-0,03)$ & $1,62(+/-0,02)$ & $1,70(+/-0,06)$ & $1,67(+/-0,03)$ \\
\hline $\operatorname{IMC}\left(\mathrm{Kg} / \mathrm{m}^{2}\right)$ & $22,60(+/-1,78)$ & $22,41(+/-1,22)$ & $24,21(+/-2,42)$ & $26,29(+/-2,53)$ \\
\hline
\end{tabular}

Fonte: Dados da pesquisa (2020).

Convém destacar que enquanto a pesquisa estava ocorrendo de forma presencial a adesão foi maior do que quando foi ajustada para estudo na forma digital. A mudança aconteceu depois da suspensão das aulas presenciais e substituição por aulas remotas mediadas pela tecnologia, a partir do decreto pela Organização Mundial de Saúde de estado de pandemia causada pela Covid-19, doença ocasionada pelo Sars-Cov-2.

Os resultados dos testes de transtornos alimentares e imagem corporal estão dispostos na tabela 2, a partir destes podese observar que $100 \%$ dos alunos apresentaram algum grau de distorção de imagem de acordo com o Body Shape 
Questionnaire (BSQ), sendo a distorção de imagem leve, a mais presente (83,7\%). Em relação aos períodos dos cursos, observou-se que dentro do curso de nutrição a distorção leve e intensa diminuíram com o decorrer da graduação, porém a distorção moderada aumentou 2,4\%. Já no curso de educação física, todos os tipos de distorção aumentaram de forma expressiva com progressão da formação. No entanto, apesar do constatado, o resultado não obteve significância estatística $(\mathrm{p}=0,606)$.

Um grupo que se acredita valorizar excessivamente a forma corporal é o de estudantes da ciência do exercício. Esta suposição é baseada em várias características relacionadas a sua matriz curricular e ambiente de educação que, em teoria, são consideradas como facilitadoras da apreciação corporal (Sundgot-borgen et al., 2021). As estudantes de Dietética e Nutrição, por sua vez, principalmente mulheres, parecem ser particularmente vulneráveis ao desenvolvimento de transtornos alimentares. Este risco aumentado pode ser devido ao seu conhecimento sobre alimentação, controle de peso e composição corporal (Mealha, Ferreira, Guerra \& Ravasco, 2013). Tradicionalmente, demonstrou-se que as mulheres se esforçam para atingir a magreza e os homens por um físico mais musculoso (Mayo \& George, 2014).

Tabela 2. Valores e Classificação dos escores do Body Shape Questionnaire, do Eating Attitudes Test (EAT-26) e da Escala de Silhueta de Stunkard de acordo com os anos de curso de uma instituição de ensino superior privada de Alagoas, de julho de 2019 a junho de 2020.

\begin{tabular}{|c|c|c|c|c|c|c|}
\hline \multirow[b]{2}{*}{ QUESTIONÁRIO } & \multicolumn{2}{|c|}{$\begin{array}{l}\text { CURSO DE } \\
\text { NUTRIÇÃO }\end{array}$} & \multicolumn{2}{|c|}{$\begin{array}{c}\text { CURSO DE } \\
\text { EDUCAÇÃO FÍSICA }\end{array}$} & \multirow[b]{2}{*}{ TOTAL } & \multirow[b]{2}{*}{ p-valor } \\
\hline & $\begin{array}{c}\text { ANOS } \\
\text { INICIAIS }\end{array}$ & $\begin{array}{c}\text { ANOS } \\
\text { FINAIS }\end{array}$ & $\begin{array}{c}\text { ANOS } \\
\text { INICIAIS }\end{array}$ & $\begin{array}{c}\text { ANOS } \\
\text { FINAIS }\end{array}$ & & \\
\hline $\begin{array}{l}\text { BSQ } \\
\text { Distorção leve }\end{array}$ & $29,1 \%$ & $22,1 \%$ & $7 \%$ & $25,6 \%$ & $83,7 \%$ & \\
\hline Distorção moderada & $2,3 \%$ & $4,7 \%$ & $2,3 \%$ & $3,5 \%$ & $12,8 \%$ & 0,606 \\
\hline Distorção grave & $\begin{array}{r}1,2 \% \\
32,6 \%\end{array}$ & $\begin{array}{r}0,0 \% \\
26,8 \%\end{array}$ & $\begin{array}{l}0,0 \% \\
9,3 \% \\
\end{array}$ & $\begin{array}{r}2,3 \% \\
31,4 \%\end{array}$ & $\begin{array}{r}3,5 \% \\
100 \%\end{array}$ & \\
\hline $\begin{array}{l}\text { EAT } \\
\text { Não indicativo para TAs }\end{array}$ & $23,3 \%$ & $25,6 \%$ & $5,8 \%$ & $23,3 \%$ & $77,9 \%$ & \\
\hline Indicativo para TAs & $9,3 \%$ & $1,2 \%$ & $3,5 \%$ & $8,1 \%$ & $\begin{array}{l}22,1 \% \\
100 \%\end{array}$ & 0,097 \\
\hline $\begin{array}{l}\text { FRS } \\
\text { Imagem corporal desejada }\end{array}$ & $17,9 \%$ & $11,9 \%$ & $1,2 \%$ & $7,1 \%$ & $38,1 \%$ & \\
\hline $\begin{array}{l}\text { Se encontra mais magro do que } \\
\text { realmente gostaria }\end{array}$ & $6,0 \%$ & $6,0 \%$ & $1,2 \%$ & $7,1 \%$ & $20,2 \%$ & 0,171 \\
\hline $\begin{array}{l}\text { Se encontra mais gordo do que } \\
\text { realmente gostaria }\end{array}$ & $9,5 \%$ & $9,5 \%$ & $7,1 \%$ & $15,5 \%$ & $\begin{array}{l}41,7 \% \\
100 \%\end{array}$ & \\
\hline
\end{tabular}

Fonte: Dados da pesquisa (2020).

Ao se analisar o Eating Attitudes Test (EAT-26) que indica tendência a comportamento de risco para transtornos alimentares, pode-se perceber que os alunos do primeiro ano de nutrição $(9,3 \%)$ apresentavam maior risco para transtornos alimentares ao comparar com os alunos do último ano (1,2\%). Esse resultado aparece de forma inversa nos alunos de educação 
física, onde os alunos dos períodos finais apresentaram maior risco $(8,1 \%)$ ao comparar com os dos períodos iniciais $(3,5 \%)$. Contudo, esse resultado não foi estatisticamente significativo $(\mathrm{p}=0,062)$.

A insatisfação corporal é uma forma de angústia relacionada ao próprio corpo que está associada a uma imagem perceptualmente distorcida de sua forma ou tamanho corporal e a uma sensação definida de desconforto (Lombardo, Cerolini, Esposito, \& Lucidi, 2021). A insatisfação com a imagem corporal entre estudantes universitários está associada a um risco aumentado de atitudes alimentares desordenadas e transtornos alimentares (Ebrahim, Alkazemi, Zafar, \& Kubow, 2019).

A faculdade pode ser um momento particularmente arriscado para o desenvolvimento de sintomas de transtornos alimentares devido aos estressores sociais únicos associados ao ambiente universitário (Quick \& Byrd-Bredbenner, 2013), por desencadear gatilhos que podem ter consequências graves aos indivíduos, tanto de ordem psicológica, quanto fisiológica. Os riscos podem ser desde uma deficiência sutil de macro ou micronutrientes ou até a morte por inanição.

Ao comparar os períodos iniciais com os finais de ambos os cursos se percebeu que os alunos de nutrição entram na graduação com maior tendência a um comportamento alimentar disfuncional e um quadro maior de distorção de imagem. Os resultados deste estudo são coerentes com os de Moraes et al. (2016) que citou a pesquisa de Reinstein et al. (1992) na qual estudantes do primeiro ano do curso de nutrição geralmente apresentam maior risco para desenvolver transtornos alimentares, sugerindo a influência positiva do conhecimento nutricional obtido ao longo da graduação como percursor de modificações favoráveis sobre as atitudes alimentares. Dado esse confirmado pelos resultados obtidos nesse estudo. No curso de Educação Física houve maior distorção de imagem entre os acadêmicos do último período, o que pode ter sido potencializado pelo culto ao corpo definido e o melhor desempenho esportivo muito desejado entre os profissionais da educação física.

Alguns estudos seccionais sobre anorexia nervosa, bulimia nervosa e transtornos de compulsão alimentar envolvendo estudantes universitários e a área da saúde vêm sendo realizados na última década, indicando prevalências muito elevadas variando de 33,3\% na área de Nutrição, 28,6\% em psicologia e 12,2\% em Educação Física (Rocha et al., 2020). Em relação a imagem corporal, pesquisas identificam uma prevalência de insatisfação em 78,2\% de acadêmicos que cursam educação física (Gonçalves, Barbosa, Rosa, \& Rodrigues, 2008). Esses dados são superiores aos resultados obtidos na população estudada.

Muitos estudos têm siso realizados com base na teoria da auto discrepância, na qual a discrepância entre a imagem corporal atual e a imagem corporal ideal é descrita como insatisfação com a imagem corporal (Mayo \& George, 2014). Estudantes universitários e atletas do sexo masculino tendem a se esforçar para obter um físico mais musculoso, o que muitas vezes se baseia em distorções na imagem corporal ideal percebida dos níveis de gordura e músculos (Ebrahim, Alkazemi, Zafar, \& Kubow, 2019).

Pela análise de distorção de imagem pela Escala de Silhueta de Stunkard, ao fazer a comparação entre períodos iniciais e finais do mesmo curso, pode-se observar que na graduação de nutrição tanto a insatisfação por excesso de peso $(9,5 \%)$ quanto por magreza $(6,0 \%)$ não apresentou variações. Porém, o resultado na graduação de educação física, mostrou o aumento expressivo de insatisfação nos dois extremos, magreza (de 1,2\% para 7,1\%) e excesso de peso (de 7,1 para $15,5 \%$ ). Apesar das constatações, esse resultado não apresentou significância estatística ( $\mathrm{p}=0,171)$.

No mais, ao realizar comparação entre os cursos de nutrição e educação física percebe-se que o curso de nutrição apresentou maior porcentagem de insatisfação em relação a magreza (12\%) do que o curso de educação física (8,3\%) e em relação a insatisfação em relação ao excesso de peso a educação física $(22,6 \%)$ apresenta maior porcentagem de insatisfação que a nutrição $(19 \%)$, com significância estatística ( $\mathrm{p}=0,024)$.

O estudo apresenta a limitação de ser transversal, não permitindo a identificação de relações de causa e efeito, além de ter tido reduzido número de participantes, provavelmente pelo estado pandêmico em que o mundo se encontrava no momento de sua realização. 
Quanto a potencialidades, o estudo realizou a comparação entre alunos no início e no final da graduação de dois cursos da área de saúde, diretamente relacionados com a estética corporal, bem como a aplicação simultânea de três questionários o que amplia a visão sobre as distorções de imagem e adoção de comportamento alimentar de risco, sendo útil como alerta da importância da abordagem de temas como transtornos alimentares e distorções de imagem de forma transversal na formação de nutricionistas e profissionais da educação física.

\section{Considerações Finais}

Os acadêmicos dos cursos de Nutrição entraram na graduação com maior tendência a um comportamento alimentar disfuncional e maior distorção de imagem, diminuindo com o decorrer dos anos de graduação; nos graduandos em Educação Física ocorreu o inverso, com maior risco de distorção de imagem e transtornos alimentares nos discentes do fim do curso, o que pode ser considerado um fenômeno preocupante.

Os achados sugerem, provavelmente, uma preocupação excessiva como um corpo "perfeito", principalmente entre discentes e profissionais das áreas de Educação Física e Nutrição. Por fim, convém ressaltar que as matrizes curriculares dos cursos da área de saúde deveriam proporcionar ampla discussão sobre os transtornos de imagem e alimentares, visando a promoção da desconstrução dos estigmas de beleza impostos pelo padrão irreal da sociedade, de modo a prevenir agravos à saúde.

Enfatiza-se a necessidade da realização de mais pesquisas que permitam maiores esclarecimentos acerta dos fatores de risco para o desenvolvimento de TAs na população acadêmica. E como a cobrança social acerca dos profissionais que atuam diretamente com questões que envolvem peso e forma corporal, pode impactar no comportamento alimentar e no agravamento desses transtornos.

\section{Referências}

American Psychiatric Association. (2014). Manual diagnóstico e estatístico de transtornos mentais: DSM-5. (5a ed.). Artmed.

Cooper, P. J., Tayloe, M. J., Cooper, Z., \& Fairburn, C. G. (1987). The development and validation of the body shape questionnaire. International Journal of Fating Disorders, 6(4), 485-494. https://doi.org/10.1002/1098-108X(198707)6:4<485::AID-EAT2260060405>3.0.CO;2-O

Di Pietro, M., \& Silveira, D. X. (2009). Internal validity, dimensionality and performance of the Body Shape Questionnaire in a group of Brazilian college students. Revista Brasileira de Psiquiatria, 31(1), 21-4. https://doi.org/10.1590/S1516-44462008005000017

Ebrahim, M., Alkazemi, D., Zafar, T. A., \& Kubow, S. (2019). Disordered eating attitudes correlate with body dissatisfaction among Kuwaiti male college students. Journal of Eating Disorders, 7(37). https://doi.org/10.1186/s40337-019-0265-Z

Fernandes, B. K. R., B., Rubim, L. J. R., Domingos M. M. A., Rios, L. F., Ferreira, J. F. R., Guimarães, M. B., \& Torres, F. Q. (2021, agosto). Principais formas de abordagem da bulimia e anorexia e suas principais consequências ao corpo humano. Brazilian Journal of Development, 7(8), 83983 - 83999. https://doi.org/10.34117/bjdv7n8-554

Garcia, C. A., Castro, T. G., \& Soares, R. M. (2010). Comportamento alimentar e imagem corporal entre estudantes de nutrição de uma universidade pública de porto alegre - RS - Revista HCPA, 30(3), 219-224.

Garner, D., Olmsted, M., Bohr, Y., \& Garfinkel, P. (1982). The Eating Attitudes Test: Psychometric features and clinical correlates. Psychological Medicine, 12(4), 871-878. https://doi.org/10.1017/s0033291700049163

Gonçalves, T. D., Barbosa, M. P., Rosa, L. C. L., \& Rodrigues, A. M. (2008). Comportamento anoréxico e percepção corporal em universitários. Jornal Brasileiro de Psiquiatria, 57(3), 166-170. https://doi.org/10.1590/S0047-20852008000300002

He, Z., Li, M., Liu, C., \& Ma, X. (2021). Relationship between body image, anxiety, food-specific inhibitory control, and emotional eating in young women with abdominal obesity: a comparative crosssectional study. Archives of Public Health, 79(11). https://doi.org/10.1186/s13690-021-00526-2

Hochman, B., Nahas, F. X., Oliveira, R. S., Filho \& Ferreira, L. M. (2005). Desenhos de pesquisa. Acta Cirúrgica Brasileira, 20(2), 02-9. https://doi.org/10.1590/S0102-86502005000800002

Kakeshita, I. S., \& Almeida, S. S. (2006). Relação entre índice de massa corporal e a percepção da autoimagem em universitários. Revista de Saúde Pública, 40(3), 497- 504. https://doi.org/10.1590/S0034-89102006000300019 
Research, Society and Development, v. 11, n. 2, e6811225418, 2022

(CC BY 4.0) | ISSN 2525-3409 | DOI: http://dx.doi.org/10.33448/rsd-v11i2.25418

Legnani, R. F. S., Legnani, E., Pereira, É. F., Gasparotto, G. S., Vieira, L. F., \& Campos, W. (2012). Transtornos alimentares e imagem corporal em acadêmicos de Educação Física - Revista de Educação Física, 18(1), 84-91. https://doi.org/10.1590/S1980-65742012000100009

Lombardo, C., Cerolini, S., Esposito, R. M., \& Lucidi, F. (2021) Psychometric properties of a Silhouette Rating Scale assessing current and ideal body size and body dissatisfaction in adults. Eating and Weight Disorders - Studies on Anorexia, Bulimia and Obesity. https://doi.org/10.1007/s40519-021-01258-6

Mayo, c., \& George v. (2014) Eating Disorder Risk and Body Dissatisfaction Based on Muscularity and Body Fat in Male University Students. Journal of American College Health, 62(6), 407-415. http://dx.doi.org/10.1080/07448481.2014.917649

Mazzaia, M. C., \& Santos, R. M. C. (2018) Fatores de risco para transtornos alimentares em graduandos de enfermagem. Acta Paulista Enfermagem, 31(5), 456-62. https://doi.org/10.1590/1982-0194201800065

Mealha, V., Ferreira, C., Guerra, I., \& Ravasco, P. (2013) Students of dietetics \& nutrition; a high risk group for eating disorders?. Nutrición Hospitalaria, 28(5), 1558-1566. https://dx.doi.org/10.3305/nh.2013.28.5.6695

Momeni, M., Ghorbani, A., \& Arjeini, Z. (2020). Disordered eating attitudes among Iranian university students of medical sciences: The role of body image perception. Nutrition and Health., 26(2), 127-133. https://doi.org/10.1177/0260106020912657

Moraes, J. M. M., Oliveira, A. C., Nunes, P. P., Lima, M. T. M. A., Abreu, J. A. O., \& Arruda, S. P. M. (2016). Fatores associados à insatisfação corporal e comportamentos de risco para transtornos alimentares entre estudantes de nutrição - Revista de Pesquisa em Saúde, 17(2), 106-111.

Penz, L. R., Bosco, S. M. D., \& Vieira, J. M. (2008). Risco para desenvolvimento de transtornos alimentares em estudantes de Nutrição - Scientia Medica, $18(3), 124-128$

Quick, V. M., \& Byrd-Bredbenner, C. (2013). Eating Disorders Examination Questionnaire (EDE-Q): norms for US college students. Eat Weight Disord, 18, 29-35. https://doi.org/10.1007/s40519-013-0015-1

Reinstein N., Koszewski WM., Chamberlin B., \& Smith-Johnson C. (1992). Prevalence of eating disorders among dietetics students: does nutrition education make a difference? Journal of the American Dietetic Association. 92(8), 949-53.

Rivas, T., Bersabé, R., Jumenez, M., \& Berrocal, C. (2010). The eating attitudes test (EAT-26) - Reliability and validity in Spanish female samples. The Spanish Journal Psychology, 13(2), 1044-1056. https://doi.org/10.1017/s1138741600002687

Rocha, G. A. F., Nogueira, J. A., Pina, N. R. L., Trindade, D. L., Ferreira, H. A. M., Biasotto, I. B., Ribeiro, C., Neto., Baptista, G. A. G., Pillar, A. B., \& Passos, S. R. L. (2020). Prevalência e fatores associados a Bulimia ou Anorexia Nervosa em universitárias da área de saúde. Brazilian Journal of Development. 6(11), 90174-90198. https://doi.org/10.34117/bjdv6n11-433

Stunkard, A., Sorensen, T., \& Schulsinger, F. (1983). Use of the Danish adption register for the study of obesity and thinness. Association for Research in Nervous and Mental Disease, 60, 115-120.

Sundgot-borgen, C., Sundgot-borgen, J. Bratland-Sanda, S., Kolle, E., Torstveit, M. K., Svantorp-Tveiten, K. M. E., \& Mathisen, T. F. (2021). Body appreciation and body appearance pressure in Norwegian university students comparing exercise science students and other students. BMC Public Health, 21, 532. https://doi.org/10.1186/s12889-021-10550-0

Zaccagni, L., Rinaldo, N., Bramanti, B., Mongillo, J., \& Gualdi-Russo, E. (2020). Body image perception and body composition: assessment of perception inconsistency by a new index. Journal of Translational Medicine, 18, 20. https://doi.org/10.1186/s12967-019-02201-1 